\title{
EXISTENCE OF AXIALLY SYMMETRIC SOLUTIONS TO THE VLASOV-POISSON SYSTEM DEPENDING ON JACOBI'S INTEGRAL*
}

\author{
ACHIM SCHULZE ${ }^{\dagger}$
}

\begin{abstract}
We prove the existence of axially symmetric solutions to the Vlasov-Poisson system in a rotating setting for sufficiently small angular velocity. The constructed steady states depend on Jacobi's integral and the proof relies on an implicit function theorem for operators.
\end{abstract}

Key words. Vlasov-Poisson system, galactic dynamics, stationary solutions

AMS 2000 subject classification: Primary: 35A05, 85A05

\section{Introduction}

In stellar dynamics, the evolution of a large ensemble of particles (e.g. stars) which interact only by their self-consistent, self-generated gravitational field, is described by the Vlasov-Poisson system

$$
\begin{array}{r}
\partial_{t} f+v \cdot \nabla_{x} f-\nabla_{x} U \cdot \nabla_{v} f=0, \\
\Delta U=4 \pi \rho, \\
\rho(t, x)=\int f(t, x, v) d v .
\end{array}
$$

Here $f=f(t, x, v) \geq 0$ is the phase-space density, where $t \in \mathbb{R}$ denotes time, and $x, v \in \mathbb{R}^{3}$ denote position and velocity. $U=U(t, x)$ is the gravitational potential of the ensemble, and $\rho=\rho(t, x)$ is its spatial density. We are looking for stationary solutions of (1.1)(1.3). The ansatz

$$
f_{0}(x, v)=\Phi(E)=\Phi\left(\frac{1}{2} v^{2}+U(x)\right)
$$

is well known, and automatically satisfies the Vlasov Equation (1.1) because the particle energy

$$
E(x, v):=\frac{1}{2} v^{2}+U(x)
$$

is a conserved quantity along characteristics. But we still have to construct the selfconsistent potential. This is done by inserting (1.4) into the Poisson equation, more precisely, we have to solve

$$
\Delta U=4 \pi h_{\Phi}(U)=4 \pi \int \Phi\left(\frac{1}{2} v^{2}+U(x)\right) d v .
$$

The ansatz (1.4) leads to spherically symmetric stationary solutions of (1.1)-(1.3), where $f$ is called spherically symmetric, iff $f(A x, A v)=f(x, v) \forall A \in O(3)$, where $O(3)$ denotes the group of orthogonal $3 \times 3$ matrices. Indeed, this is a special case of a

\footnotetext{
*Received: March 13, 2008; accepted (in revised version): July 17, 2008. Communicated by Pierre Degond.

${ }^{\dagger}$ Mathematisches Institut der Universität Bayreuth D 95440 Bayreuth, Germany (achim.schulze@yahoo.de).
} 
more general result of Gidas, $\mathrm{Ni}$ and Nirenberg [2]. If one is interested in stationary solutions with less symmetry, more invariants can be added to (1.4), so that the right-hand side of (1.5) explicitly depends on $x$.

One possibility is to consider a rotating system. If the ensemble is rotating about a given axis, say the $x_{3}$-axis, we can change to a rotating frame by changing coordinates as follows:

$$
\zeta:=R_{t} x, \quad \eta:=R_{t} v-\Omega \times\left(R_{t} x\right)
$$

where

$$
R_{t}:=\left(\begin{array}{ccc}
\cos (\omega t) & \sin (\omega t) & 0 \\
-\sin (\omega t) & \cos (\omega t) & 0 \\
0 & 0 & 1
\end{array}\right), \quad \Omega:=\left(\begin{array}{l}
0 \\
0 \\
\omega
\end{array}\right)
$$

and the (rotational) velocity $\omega>0$ is given. The Vlasov-Poisson system then takes the form

$$
\begin{gathered}
\partial_{t} f+\eta \cdot \nabla_{\zeta} f-\left(\nabla_{\zeta} U+\Omega \times(\Omega \times \zeta)+2(\Omega \times \eta)\right) \cdot \nabla_{\eta} f=0, \\
\Delta_{\zeta} U(t, \zeta)=4 \pi \rho(t, \zeta), \\
\rho(t, \zeta)=\int f(t, \zeta, \eta) d \eta
\end{gathered}
$$

and the characteristic system of the Vlasov Equation (1.6) is

$$
\left\{\begin{array}{l}
\dot{\zeta}=\eta \\
\dot{\eta}=-\partial_{\zeta} U(t, \zeta)-2 \Omega \times \eta-\Omega \times(\Omega \times \zeta) .
\end{array}\right.
$$

This system has the following expression as a conserved quantity, if $U$ is timeindependent,

$$
E_{J}:=\frac{1}{2} \eta^{2}+U(\zeta)-\frac{1}{2}|\Omega \times \zeta|^{2},
$$

where $E_{J}$ is also called Jacobi's integral. A natural ansatz for the construction of stationary solutions of (1.6)-(1.8) is now

$$
f(\zeta, \eta)=\varphi\left(E_{J}\right)=\varphi\left(\frac{1}{2}|\eta|^{2}+U(\zeta)-\frac{1}{2} \omega^{2} r^{2}\right),
$$

for a suitable function $\varphi: \mathbb{R} \rightarrow \mathbb{R}^{+}$, where $r:=r(x)=\sqrt{\zeta_{1}^{2}+\zeta_{2}^{2}}$. In the original coordinates, $(x, v)$ one can easily verify that this ansatz leads to

$$
g(x, v):=f(\zeta, \eta)=\varphi\left(\frac{1}{2} v^{2}+U\left(R_{t} x\right)-\omega P\right),
$$

where we define $P$ to be the third component of the angular momentum, that is $P:=x_{1} v_{2}-x_{2} v_{1}$, which is a conserved quantity of the characteristic system of the Vlasov Equation (1.1) if $U$ is axially symmetric with respect to the $x_{3}$-axis. Obviously, 
the function $f=f(\zeta, \eta)$ then automatically satisfies (1.6), and one has to solve the Poisson equation, where we relabel $\zeta$ and $\eta$ to $x$ and $v$, respectively,

$$
\Delta U=\int \varphi\left(\frac{1}{2} v^{2}+U(x)-\frac{1}{2} \omega^{2} r^{2}\right) d v=: \tilde{h}(\omega, r(x), U(x)) .
$$

So if we construct an axially symmetric $U$ that solves (1.10), the corresponding functions $(g, U)$, with $g$ defined as above, will also be a stationary solution of (1.1)-(1.3). Clearly, our ansatz for $f$ satisfies (1.6) without any symmetry assumptions on $U$, and this gives hope for the construction of stationary solutions with less symmetry, for example, triaxial solutions. Here, triaxial means that the three half axes of the support of the spatial density have pairwise different length. In particular, a triaxial solution is not axially symmetric.

Equation (1.10) has been studied, by Vandervoort [9], among others. He observed numerically that if $\varphi$ is of the form

$$
\varphi\left(E_{J}\right)=\left(E_{0}-E_{J}\right)_{+}^{\beta-3 / 2},
$$

then for $0.5<\beta \leq 0.808$ there are triaxial solutions to (1.10) for sufficiently large $\omega$. For small $\omega$, or $\beta>0.808$, all numerically constructed solutions are axially symmetric. Consequently, (1.10) seems to be of particular interest for the construction of ellipsoidal solutions, but to our knowledge no self-consistent ellipsoidal systems to (1.1)-(1.3) or (1.6)-(1.8) have been constructed analytically yet.

We will prove that there exist axially symmetric solutions to (1.10) for small $\omega$ under suitable assumptions on $\varphi$, where we treat the case $\beta>5 / 2$ in (1.11). For this purpose, we require that for $\omega=0$, we have a nontrivial, spherically symmetric solution $\left(f_{0}, U_{0}\right)$ of $(1.10)$. Note that in this case the right hand side of (1.10) only depends on $U_{0}$. For $\omega \neq 0$, we want to apply an implicit function theorem to get solutions, which arise by deforming $U_{0}$, where certain symmetries are conserved. The central idea that makes this approach work is to look for a solution $U^{\omega}$ as a deformation of $U_{0}$, i.e., $U^{\omega}=U_{0}(\gamma(x))$ for some diffeomorphism $\gamma$ on $\mathbb{R}^{3}$, and to formulate the problem in terms of finding zeros of a suitable operator $T$ over the space of such deformations instead of the space of the potentials. While the original problem (1.10) had to be solved in $\mathbb{R}^{3}$, we will only need to know the deformation on a compact neighborhood of the support of the original solution $\left(f_{0}, \rho_{0}, U_{0}\right)$, which provides useful compactness properties. Furthermore, finite radius and finite mass of the constructed solutions are just consequences of the corresponding properties of $\left(f_{0}, \rho_{0}, U_{0}\right)$.

The machinery presented here shows great promise for the construction of triaxial solutions, since the allowed perturbations for the potential $U_{0}$ only have mirror symmetry, and thus would match a triaxial setup. But up to now we have no method to exclude axial symmetry with respect to the $x_{3}$-axis for the perturbations constructed by the implicit function theorem.

The approach described above has been used by Lichtenstein for proving the existence of slowly rotating Newtonian stars, as described by selfgravitating fluid balls $[4,5]$. A translation of Lichtenstein's approach into modern mathematical language is due to Heilig [3].

The investigations made there were applied to the Vlasov-Poisson system in [8], where stationary solutions to (1.1)-(1.3) of the form $f(x, v)=\varphi(E) \psi(\omega P)$ were constructed. There, the potential $U$ was a priori axially symmetric, so that the expression $P=x_{1} v_{2}-x_{2} v_{1}$ is a conserved quantity with respect to the characteristic system. The procedure described there is the basis of our approach. 
This paper is organized as follows: In the next section we rewrite the problem in terms of finding zeros of the operator $T$, then we state the main result and prove it using an implicit function theorem. For this, we need certain properties of $T$ which can be proved as in [8], except some minor technical modifications and one lemma, where the symmetry of the allowed perturbations enters in. In section 3, we generalize this important lemma to mirror symmetry.

\section{The main result}

The mappings, which leave our solutions invariant, are in the set

$$
\begin{aligned}
S:=\left\{\tau_{110}:\left(x_{1}, x_{2}, x_{3}\right)\right. & \mapsto\left(x_{1}, x_{2},-x_{3}\right), \tau_{101}:\left(x_{1}, x_{2}, x_{3}\right) \mapsto\left(x_{1},-x_{2}, x_{3}\right), \\
& \left.\tau_{011}:\left(x_{1}, x_{2}, x_{3}\right) \mapsto\left(-x_{1}, x_{2}, x_{3}\right)\right\} .
\end{aligned}
$$

Now let $B_{R}:=\left\{x \in \mathbb{R}^{3}|| x \mid \leq R\right\}$ and define

$$
C_{S}\left(B_{R}\right):=\left\{f \in C\left(B_{R}\right) \mid f(A x)=f(x), A \in S, x \in B_{R}\right\} .
$$

Then we have

$$
\nabla f(0)=0, \quad \text { if } \quad f \in C^{1}\left(B_{R}\right) \cap C_{S}\left(B_{R}\right) .
$$

For $\varphi: \mathbb{R} \rightarrow[0, \infty[$ we require

$(\varphi 1) \varphi \in C^{1}(\mathbb{R})$, there is $E_{0} \in \mathbb{R}$ with $\varphi\left(E_{J}\right)=0$ for $E_{J} \geq E_{0}$, and $\varphi\left(E_{J}\right)>0$ for $E_{J}<$ $E_{0}$.

$(\varphi 2) \varphi$ is strictly decreasing in $]-\infty, E_{0}[$.

$(\varphi 3)$ The ansatz $f_{0}(x, v)=\varphi\left(E_{J}\right)$ with $\omega=0$ produces a nontrivial, spherically symmetric solution $\left(f_{0}, \rho_{0}, U_{0}\right)$ of $(1.1)-(1.3)$ with $\rho_{0} \in C_{0}^{1}\left(\mathbb{R}^{3}\right)$, supp $\rho_{0}=B_{1}$ and $U_{0} \in C^{2}\left(\mathbb{R}^{3}\right)$ with $\lim _{|x| \rightarrow \infty} U_{0}(x)=0$.

Examples for a functions satisfying $(\varphi 1)-(\varphi 3)$ are the so-called polytropes,

$$
\varphi\left(E_{J}\right):=\left(E_{0}-E_{J}\right)_{+}^{k},
$$

for $k>1$ and suitable $E_{0}<0$. Now we can state the main theorem.

Theorem 2.1. Let $r:=\sqrt{x_{1}^{2}+x_{2}^{2}}$. There exists $\omega_{0}>0$, such that for all $\left.\omega \in\right]-\omega_{0}, \omega_{0}[$ there exits a nontrivial solution $\left(f^{\omega}, \rho^{\omega}, U^{\omega}\right)$ of (1.6)-(1.8) with

(i) $f^{\omega}(x, v)= \begin{cases}\varphi\left(\frac{1}{2} v^{2}+U^{\omega}(x)-\frac{1}{2} \omega^{2} r^{2}\right) & \text { for }|x|<4 \\ 0 & \text { otherwise }\end{cases}$

(ii) $\left(f^{0}, \rho^{0}, U^{0}\right)=\left(f_{0}, \rho_{0}, U_{0}\right)$ and for $|\omega|<\omega_{0},\left(f^{\omega}, \rho^{\omega}, U^{\omega}\right)$ has the following symmetry properties: For all $A \in S$ we have

$$
f^{\omega}(A x, A v)=f^{\omega}(x, v), \quad \rho^{\omega}(A x)=\rho(x), \quad U^{\omega}(A x)=U^{\omega}(x)
$$

and $\left(f^{\omega}, \rho^{\omega}, U^{\omega}\right)$ is not spherically symmetric for $\omega \neq 0$.

(iii) $\rho^{\omega} \in C_{c}^{1}\left(\mathbb{R}^{3}\right)$ and $U^{\omega} \in C_{b}^{2}\left(\mathbb{R}^{3}\right)$, where $\rho^{\omega}(x)=\int f^{\omega}(x, v) d v$.

(iv) The mappings $]-\omega_{0}, \omega_{0}\left[\ni \omega \mapsto \rho^{\omega}\right.$ and $]-\omega_{0}, \omega_{0}\left[\ni \omega \mapsto U^{\omega}\right.$ are continuous with respect to the norms $\|\cdot\|_{1, \infty}$ or $\|\cdot\|_{2, \infty}$, respectively, where we defined $\|\cdot\|_{1, \infty}:=$ $\|\cdot\|_{\infty}+\|\nabla \cdot\|_{\infty}$ and the norm $\|\cdot\|_{2, \infty}$ is defined analogously. 
REMARK 2.1. If we add rotations about the $x_{3}$-axis to the set $S$, the proof of Theorem 2.1 still holds - we can essentially follow the proof given here, and this shows that the constructed solutions in Theorem 2.1 have to be axially symmetric a posteriori. This follows by the uniqueness of the mapping given by the implicit function theorem (Theorem A.1).

For the proof of Theorem 2.1, we require the following lemmas.

LEMMA 2.2. The spherically symmetric solution $\left(f_{0}, \rho_{0}, U_{0}\right)$ has the following properties.

(a) The potential $U_{0}$ is given by

$$
U_{0}(x)=-\int \frac{\rho_{0}(y)}{|x-y|} d y=-\frac{4 \pi}{|x|} \int_{0}^{|x|} s^{2} \rho_{0}(s) d s-4 \pi \int_{|x|}^{\infty} s \rho_{0}(s) d s, x \in \mathbb{R}^{3} .
$$

(b) $\rho_{0}$ is decreasing with $\rho_{0}(0)>0, U_{0}^{\prime \prime}(0)>0$ and for every $R>0$ there exists $C>0$ such that $U_{0}^{\prime}(|x|) \geq C|x|,|x| \in[0, R]$, and $U_{0}(1)=E_{0}$.

(c) $\rho_{0}^{\prime}$ is Hölder continuous and $U_{0}^{\prime} \in C^{2}\left(\dot{\mathbb{R}}^{3}\right)$, where $\dot{\mathbb{R}}^{3}:=\mathbb{R}^{3} \backslash\{0\}$.

Proof. The formula

$$
U_{0}^{\prime}(|x|)=\frac{4 \pi \int_{0}^{|x|} s^{2} \rho_{0}(s) d s}{|x|^{2}}
$$

easily follows from the Poisson equation with spherical symmetry, and since we require $\lim _{|x| \rightarrow \infty} U_{0}(x)=0$, the representation for $U_{0}$ holds by uniqueness. As to (b), for $\omega=0$ we have $f_{0}(x, v)=f_{0}(E)=f_{0}\left(\frac{1}{2} v^{2}+U_{0}(x)\right)$, and this implies

$$
\rho_{0}(x)=\int_{\mathbb{R}^{3}} f_{0}(x, v) d v=h_{0}\left(U_{0}(x)\right):=4 \pi \sqrt{2} \int_{U_{0}(x)}^{E_{0}} \varphi(E) \sqrt{E-U_{0}(x)} d E,
$$

where the function $h$ is continuously differentiable, and with $(\varphi 1),(\varphi 2)$, we have $h^{\prime}(s)<0$ for $s<E_{0}$. Consequently, $\rho_{0}$ is decreasing because $U_{0}$ is increasing and since the steady state $\left(f_{0}, U_{0}\right)$ is assumed to be nontrivial, we must have $\rho_{0}(0)>0$. Thus $U_{0}^{\prime}(|x|)>0,|x|>0$, and since $U_{0}^{\prime \prime}(0)=(4 \pi / 3) \rho_{0}(0)>0$ this implies the estimate on $U_{0}^{\prime}$ from above. The assertion that $U_{0}(1)=E_{0}$ follows from (2.2) and the assumption $\operatorname{supp} \rho_{0}=B_{1}$. The regularity of $U_{0}^{\prime}$ follows from the formula for $U_{0}^{\prime}$ above and the fact that $\rho_{0} \in C_{c}^{1}$, which we deduce again from (2.2). Finally, the proof of the Hölder continuity of $\rho_{0}^{\prime}$ will be part of the next Lemma.

Lemma 2.3. Let $E_{1}:=U_{0}(2)-E_{0}$ and define $f$ by

$$
f(x, v)= \begin{cases}\varphi\left(\frac{1}{2} v^{2}+U(x)-\frac{1}{2} \omega^{2} r^{2}\right) & \text { for } U(x)<E_{0}+E_{1}, \\ 0 & \text { otherwise }\end{cases}
$$

where $\varphi$ satisfies $(\varphi 1),(\varphi 2)$ and $U \in C_{b}^{2}\left(\mathbb{R}^{3}\right)$, with $U(x)>E_{0}+E_{1}$ for $|x|>4$. Then the following holds:

$$
\begin{aligned}
\rho_{f}(x) & :=\int_{\mathbb{R}^{3}} f(x, v) d v \\
& =\tilde{h}(\omega, r(x), U(x)) \\
& = \begin{cases}h\left(U(x)-\frac{1}{2} \omega^{2} r^{2}\right) & \text { for } U(x)<E_{0}+E_{1}, \\
0 & \text { otherwise, }\end{cases}
\end{aligned}
$$


with

$$
h(s)=4 \pi \sqrt{2} \int_{s}^{E_{0}} \sqrt{E-s} \varphi(E) d E .
$$

Furthermore, $\tilde{h} \in C^{1}(\mathbb{R} \times[0, \infty[\times \mathbb{R})$ and for every bounded set $B \subset \mathbb{R} \times[0, \infty[\times \mathbb{R}$ there are constants $C>0$ and $\mu \in] 0,1\left[\right.$ such that for $(\omega, r, u),\left(\omega^{\prime}, r, u^{\prime}\right) \in B$ we have

$$
\begin{aligned}
& \left|\partial_{r} \tilde{h}(\omega, r, u)\right| \leq C r, \\
& \left|\tilde{h}(\omega, r, u)-\tilde{h}\left(\omega^{\prime}, r, u^{\prime}\right)\right| \leq C\left(\left|\omega-\omega^{\prime}\right| r+\left|u-u^{\prime}\right|\right), \\
& \left|\partial_{u} \tilde{h}(\omega, r, u)-\partial_{u} \tilde{h}\left(\omega^{\prime}, r, u^{\prime}\right)\right| \leq C\left(\left|\omega-\omega^{\prime}\right|+\left|u-u^{\prime}\right|^{\mu}\right) .
\end{aligned}
$$

In addition, for $\omega=0$, the function $\tilde{h}(0, \cdot, \cdot)$ does not depend on $r(x)$ and we can write $h_{0}:=\tilde{h}(0,0, u)$.

Proof. Introducing polar coordinates, we have for $U(x)<E_{0}+E_{1}$,

$$
\begin{aligned}
\rho(x) & =\int \varphi\left(\frac{1}{2} v^{2}+U(x)-\frac{1}{2} \omega^{2} r^{2}\right) d v \\
& =4 \pi \int_{0}^{\infty} t^{2} \varphi\left(\frac{1}{2} t^{2}+U(x)-\frac{1}{2} \omega^{2} r^{2}\right) d t \\
& =4 \pi \sqrt{2} \int_{U(x)-\frac{1}{2} \omega^{2} r^{2}}^{E_{0}}\left(E-U(x)+\frac{1}{2} \omega^{2} r^{2}\right)^{1 / 2} \varphi(E) d E
\end{aligned}
$$

and (2.3) follows.

We have $h \in C^{1}(\mathbb{R})$ with

$$
h^{\prime}(s)=-4 \pi \sqrt{2} \int_{s}^{E_{0}} \frac{1}{2 \sqrt{E-s}} \varphi(E) d E
$$

for $s<E_{0}$ and $h^{\prime}(s)=0$ for $s \geq E_{0}$ and the first two estimates follow. Next,

$$
\begin{aligned}
h^{\prime \prime}(s) & =-4 \pi \sqrt{2} \frac{d}{d s} \int_{0}^{E_{0}-s} \frac{1}{2 \sqrt{E}} \varphi(E+s) d E \\
& =-4 \pi \sqrt{2} \int_{0}^{E_{0}-s} \frac{1}{2 \sqrt{E}} \varphi^{\prime}(E+s) d E \\
& =-4 \pi \sqrt{2} \int_{s}^{E_{0}} \frac{1}{2 \sqrt{E-s}} \varphi^{\prime}(E) d E
\end{aligned}
$$

yields local Lipschitz continuity of $\partial_{u} \tilde{h}$ with respect to $\omega$ and $u$, and the proof is complete.

We want to find solutions of the equation

$$
\Delta U=4 \pi \tilde{h}(\omega, r(x), U) .
$$

The main idea is to rewrite problem (2.4) in terms of finding the zeros of an operator $T$, which does not act directly on the space of potentials, but on deformations of the 
given spherically symmetric potential $U_{0}$. We define the following Banach spaces, which will serve as the domain and range of $T$. We define $X$ by

$$
\begin{aligned}
& X:=\left\{f \in C_{S}\left(B_{4}\right)\left|f(0)=0, f \in C^{1}\left(\dot{B}_{4}\right), \exists C>0:\right| \nabla f(x) \mid \leq C, x \in \dot{B}_{4},\right. \\
&\left.\forall x \in \partial B_{1}: \lim _{t \rightarrow 0, t>0} \nabla f(t x)=: \nabla f(0 x) \text { exists, uniformly in } x \in \partial B_{1}\right\},
\end{aligned}
$$

where $\partial B_{1}:=\left\{x \in \mathbb{R}^{3}|| x \mid=1\right\}$ and $\dot{B}_{4}:=B_{4} \backslash\{0\}$. We equip $X$ with the norm

$$
\|f\|_{X}:=\sup _{x \in \dot{B}_{4}}|\nabla f(x)|, \quad f \in X .
$$

We define $Y$ by

$$
\begin{aligned}
Y:=\{ & f \in C_{S}\left(B_{4}\right)\left|f(0)=0, f \in C^{1}\left(B_{4}\right), \exists C>0:\right| \nabla f(x)|\leq C| x \mid, x \in B_{4}, \\
& \left.\forall x \in \partial B_{1}: \lim _{t \rightarrow 0, t>0} \frac{\nabla f(t x)}{t}=: \frac{\nabla f(0 x)}{0} \text { exists, uniformly in } x \in \partial B_{1}\right\}
\end{aligned}
$$

with norm

$$
\|f\|_{Y}:=\sup _{x \in \dot{B}_{4}} \frac{|\nabla f(x)|}{|x|}, \quad f \in Y .
$$

For example, we have $|x| \in X$ and $|x|^{2} \in Y$, but $|x| \notin Y$.

To explain more precisely, how we will use functions in $X$ to deform the potential $U_{0}$, we need the following lemma.

Lemma 2.4. For $\zeta \in X$, let

$$
g_{\zeta}: B_{4} \rightarrow \mathbb{R}^{3}, \quad g_{\zeta}(x):=x+\zeta(x) \frac{x}{|x|}, \quad x \in \dot{B}_{4}, \quad g_{\zeta}(0)=0 .
$$

Then there exists $r>0$ such that for all $\zeta \in \Omega$ where

$$
\Omega:=\left\{\zeta \in X \mid\|\zeta\|_{X}<r\right\}
$$

we have:

(a) $g_{\zeta}: B_{4} \rightarrow B_{4, \zeta}:=g_{\zeta}\left(B_{4}\right) \quad$ is a homeomorphism, $g_{\zeta}: \dot{B}_{4} \rightarrow \dot{B}_{4, \zeta} \quad$ is $\quad$ a $\quad C^{1}$ diffeomorphism with

$$
\left|D g_{\zeta}(x)-\mathrm{id}\right|<\frac{1}{2}, \quad x \in \dot{B}_{4}
$$

and for every $x \in \partial B_{1}$ the mapping

$$
g_{\zeta}: \overline{0,4 x} \ni y \mapsto g_{\zeta}(y) \in \overline{0,\left|g_{\zeta}(4 x)\right| x}
$$

is one-to-one, onto and preserves the natural ordering of points in $\overline{0,4 x}$, where $\overline{x_{1}, x_{2}}:=\left\{x_{1}+\lambda\left(x_{2}-x_{1}\right) \mid \lambda \in[0,1]\right\}$ for $x_{1}, x_{2} \in \mathbb{R}^{3}$.

(b) $\frac{1}{2}|x| \leq\left|g_{\zeta}(x)\right| \leq \frac{3}{2}|x|, x \in B_{4}$, and $g_{\zeta}\left(B_{2}\right) \subset \stackrel{\circ}{B}_{3}, B_{3} \subset g_{\zeta}\left(B_{4}\right) \subset B_{5}$

(c) $g_{\zeta}(A x)=A g_{\zeta}(x), x \in B_{4}$ and $g_{\zeta}^{-1}(A x)=A g_{\zeta}^{-1}(x), x \in B_{4, \zeta}, A \in S$ 
(d) $\left|D g_{\zeta}^{-1}(x)-\mathrm{id}\right|<\frac{1}{2}, x \in \dot{B}_{4, \zeta}$, and there exists a constant $C>0$ such that for all $\zeta, \zeta^{\prime} \in \Omega$ :

$$
\frac{1}{|x|}\left|g_{\zeta}(x)-g_{\zeta^{\prime}}(x)\right|+\left|D g_{\zeta}(x)-D g_{\zeta^{\prime}}(x)\right| \leq C\left\|\zeta-\zeta^{\prime}\right\|_{X}, \quad x \in \dot{B}_{4}
$$

and

$$
\left|g_{\zeta}^{-1}(x)-g_{\zeta^{\prime}}^{-1}(x)\right| \leq C\left\|\zeta-\zeta^{\prime}\right\|_{X}|x|, \quad x \in B_{3}
$$

Proof. In $\dot{B}_{4}$, we have for $i, j=1,2,3$ that

$$
\partial_{x_{i}} g_{\zeta, j}(x)=\delta_{i j}+\partial_{x_{i}} \zeta(x) \frac{x_{j}}{|x|}+\frac{\zeta(x)}{|x|}\left(\delta_{i j}-\frac{x_{i} x_{j}}{|x|^{2}}\right),
$$

and therefore

$$
\left|D g_{\zeta}(x)-\mathrm{id}\right|<3\|\zeta\|_{X}
$$

Using the inverse function theorem, the first two assertions in (a) follow. For $x \in \partial B_{1}$,

$$
g_{\zeta}(t x)=t x+\zeta(t x) x=x(t+\zeta(t x))
$$

and

$$
\frac{d}{d t}(t+\zeta(t x))=1+\nabla \zeta(t x) \cdot x>0, \quad \text { for }\|\zeta\|_{X} \text { small }
$$

and the proof of (a) is complete.

We have $|\zeta(x)| \leq\|\zeta\|_{X}|x|$ for $x \in B_{4}$, which implies (b) for $r>0$ sufficiently small. Assertion (c) is easily verified as well. If we choose an even smaller $r$, we also have the first claim of (d), because

$$
D g_{\zeta}^{-1}(x)=\left(D g_{\zeta}\right)^{-1}\left(g_{\zeta}^{-1}(x)\right)
$$

The estimate for $g_{\zeta}-g_{\zeta^{\prime}}$ follows from the definition of $g_{\zeta}$, and the estimate for $D g_{\zeta}-$ $D g_{\zeta^{\prime}}$ follows from (2.5).

For $x \in \dot{B}_{3}$, we have from (b) that $x \in g_{\zeta}\left(B_{4}\right) \cap g_{\zeta^{\prime}}\left(B_{4}\right)$. Consequently, there exists $y \in \dot{B}_{4}$ with $x=g_{\zeta^{\prime}}(y)$. Now we have

$$
\begin{aligned}
\left|g_{\zeta}^{-1}(x)-g_{\zeta^{\prime}}^{-1}(x)\right| & =\left|g_{\zeta}^{-1}\left(g_{\zeta^{\prime}}(y)\right)-y\right| \\
& =\left|g_{\zeta}^{-1}\left(g_{\zeta^{\prime}}(y)\right)-g_{\zeta}^{-1}\left(g_{\zeta}(y)\right)\right| \\
& \leq 2\left|g_{\zeta}(y)-g_{\zeta^{\prime}}(y)\right| \leq 2\left\|\zeta-\zeta^{\prime}\right\|_{X}|y| \\
& \leq 4\left\|\zeta-\zeta^{\prime}\right\|_{X}|x|,
\end{aligned}
$$

where we used the mean value theorem, the estimate for $D g_{\zeta}^{-1}$, and $\overline{g_{\zeta}(y), g_{\zeta^{\prime}}(y)} \subset$ $g_{\zeta}\left(\dot{B}_{4}\right)$.

We want to find solutions of (2.4) such that

$$
U(x)=U_{\zeta}(x):=U_{0}\left(g_{\zeta}^{-1}(x)\right), \quad x \in B_{4, \zeta},
$$


with a suitable $\zeta \in \Omega$. Obviously, we need $U$ to be defined on the whole space $\mathbb{R}^{3}$, but this is only a technical problem. We use the fundamental solution of the Poisson equation to integrate (2.4) and we then have to solve

$$
U_{0}(x)+\int_{B_{4, \zeta}} \frac{\tilde{h}\left(\omega, r(y), U_{0}\left(g_{\zeta}^{-1}(y)\right)\right)}{\left|g_{\zeta}(x)-y\right|} d y=0, \quad x \in B_{4} .
$$

This equation essentially contains the operator we are looking for, but we have to make some modifications. We also want to get rid of the dependence on $\zeta$ in the integration domain.

Proof. [Proof of Theorem 2.1] For $\zeta \in \Omega$ and $\omega \in \mathbb{R}$, we define

$$
\begin{aligned}
T(\omega, \zeta)(x): & =U_{0}(x)+\int_{B_{3}} \frac{\tilde{h}\left(\omega, r(y), U_{0}\left(g_{\zeta}^{-1}(y)\right)\right)}{\left|g_{\zeta}(x)-y\right|} d y \\
& -U_{0}(0)-\int_{B_{3}} \frac{\tilde{h}\left(\omega, r(y), U_{0}\left(g_{\zeta}^{-1}(y)\right)\right)}{|y|} d y, \quad x \in B_{4} .
\end{aligned}
$$

Suppose we already know that this defines a continuous operator

$$
T:]-\tilde{\omega}, \tilde{\omega}[\times \Omega \rightarrow Y,
$$

for some $\tilde{\omega}>0$, and $T$ is continuously Frechet-differentiable with respect to $\zeta$, where

$$
\partial_{\zeta} T(0,0): X \rightarrow Y
$$

is an isomorphism. The first two assertions follow from [8, Sec. 2], and the last assertion will be verified in section 3 . Also in section 3 , the symmetry of the perturbations will play a crucial role.

The definition of $Y$ requires that $T(\omega, \zeta)(0)=0$ which explains why we substracted the constant in (2.7). Due to assumption $(\varphi 3)$, we know that $T(0,0)=0$, because $g_{0}=$ id and $\operatorname{supp} \rho_{0}=\operatorname{supp} h_{0} \circ U_{0}=B_{1} \subset B_{3}$. The implicit function theorem [1, Thm 15.1], also stated in the Appendix of this article as Theorem A.1, now guarantees the existence of $\left.\omega_{1} \in\right] 0, \tilde{\omega}[$ and the existence of a continuous mapping

$$
]-\omega_{1}, \omega_{1}\left[\ni \omega \mapsto \zeta^{\omega} \in \Omega\right.
$$

such that

$$
\left.T\left(\omega, \zeta^{\omega}\right)=0, \quad \omega \in\right]-\omega_{1}, \omega_{1}[
$$

and $\zeta^{0}=0$. We also will require that $\omega^{2} r^{2}<E_{1}$ in $B_{4}$, where $E_{1}$ is defined in Lemma 2.3 , and therefore we define

$$
\omega_{0}:=\min \left\{\omega_{1}, \frac{\sqrt{\left|E_{1}\right|}}{4}\right\}
$$

Now let $\zeta=\zeta^{\omega}$, where we choose a fixed $\left.\omega \in\right]-\omega_{0}, \omega_{0}[$ and define

$$
\rho_{\zeta}(x):=\tilde{h}\left(\omega, r(x), U_{0}\left(g_{\zeta}^{-1}(x)\right)\right), \quad x \in B_{3} .
$$

Then we have $\rho_{\zeta} \in C_{S}\left(B_{3}\right) \cap C^{1}\left(\dot{B}_{3}\right)$. By Lemma 2.3, $\rho_{\zeta}>0$ at most, if $U_{0}\left(g_{\zeta}^{-1}(x)\right)<$ $E_{0}+E_{1}$, which is equivalent to $\left|g_{\zeta}^{-1}(x)\right|<2$ by Lemma 2.2 . Consequently,

$$
\operatorname{supp} \rho_{\zeta}=g_{\zeta}\left(B_{2}\right) \subset \stackrel{\circ}{B}_{3} .
$$


We extend $\rho_{\zeta}$ by 0 to all of $\mathbb{R}^{3}$, and we achieve

$$
\rho_{\zeta} \in C_{c}\left(\mathbb{R}^{3}\right), \quad \operatorname{supp} \rho_{\zeta} \subset \stackrel{\circ}{B}_{3} .
$$

We want equation (2.9) to hold everywhere, but we have not defined $g_{\zeta}$ globally.

We can rewrite $T(\omega, \zeta)=0$ as

$$
U_{0}(x)=-\int_{B_{3}} \frac{\rho_{\zeta}(y)}{\left|g_{\zeta}(x)-y\right|} d y+C, \quad x \in B_{4},
$$

or

$$
U_{0}\left(g_{\zeta}^{-1}(x)\right)=-\int_{B_{3}} \frac{\rho_{\zeta}(y)}{|x-y|} d y+C, \quad x \in B_{4, \zeta},
$$

where

$$
C:=U_{0}(0)+\int_{B_{3}} \frac{\rho_{\zeta}(y)}{|y|} d y
$$

Now define

$$
U_{\zeta}(x):=-\int_{\mathbb{R}^{3}} \frac{\rho_{\zeta}(y)}{|x-y|} d y+C .
$$

Then we have $U_{\zeta} \in C^{1}\left(\mathbb{R}^{3}\right)$, with

$$
U_{\zeta}(x)=U_{0}\left(g_{\zeta}^{-1}(x)\right), \quad x \in B_{3} \subset B_{4, \zeta},
$$

and thus $\rho_{\zeta} \in C_{c}^{1}\left(\mathbb{R}^{3}\right)$ and $U_{\zeta} \in C_{b}^{2}\left(\mathbb{R}^{3}\right)$ with $\Delta U_{\zeta}=4 \pi \rho_{\zeta}$ in $\mathbb{R}^{3}$.

Furthermore,

$$
\Delta U_{\zeta}=4 \pi \tilde{h}\left(\omega, r(x), U_{\zeta}(x)\right), \quad x \in B_{3} \subset B_{4, \zeta} .
$$

The last equation holds even in $\mathbb{R}^{3}$. We have to show that

$$
\rho_{\zeta}(x)=\tilde{h}\left(\omega, r(x), U_{\zeta}(x)\right), \quad x \in \mathbb{R}^{3},
$$

that is, $U_{\zeta}(x)>E_{0}+E_{1}$ for $x \in \mathbb{R}^{3} \backslash g_{\zeta}\left(B_{2}\right)$. We know that

$$
\Delta U_{\zeta}(x)=0, \quad x \in \mathbb{R}^{3} \backslash g_{\zeta}\left(B_{2}\right),
$$

$\lim _{|x| \rightarrow \infty} U_{\zeta}(x)=C$, and

$$
\begin{aligned}
& U_{\zeta}(x)=E_{0}+E_{1}, \quad x \in \partial g_{\zeta}\left(B_{2}\right), \\
& U_{\zeta}(x)>E_{0}+E_{1}, \quad x \in B_{3} \backslash g_{\zeta}\left(B_{2}\right) .
\end{aligned}
$$

Here we used (2.10) and the monotonicity of $U_{0}(|x|)$ with $U_{0}(2)=E_{0}+E_{1}$. If $C \leq$ $E_{0}+E_{1}$, we have a contradiction of the maximum principle. Therefore, $C>E_{0}+E_{1}$ and again by the maximum principle, $U_{\zeta}>E_{0}+E_{1}$ on $\mathbb{R}^{3} \backslash g_{\zeta}\left(B_{2}\right)$ and consequently (2.11) holds in $\mathbb{R}^{3}$.

Now define $\rho^{\omega}:=\rho_{\zeta}, U^{\omega}:=U_{\zeta}$ and

$$
\begin{aligned}
f^{\omega}(x, v) & := \begin{cases}\varphi\left(\frac{1}{2} v^{2}+U^{\omega}(x)-\frac{1}{2} \omega^{2} r^{2}\right), & \text { for } U^{\omega}(x)<E_{0}+E_{1}, \\
0 & \text { otherwise },\end{cases} \\
& = \begin{cases}\varphi\left(\frac{1}{2} v^{2}+U^{\omega}(x)-\frac{1}{2} \omega^{2} r^{2}\right), & \text { for }|x|<4, \\
0 & \text { otherwise, } .\end{cases}
\end{aligned}
$$


$f^{\omega}$ defined by (2.12) solves the Vlasov Equation (1.6) because it is constant along characteristics. More precisely, we have $U_{\zeta}(x)-\frac{1}{2} \omega^{2} r^{2}>E_{0}$ in a neighborhood of $\partial B_{4}$, if we choose $\omega_{0}$ sufficiently small as in (2.8). If we then fix $(x, v)$ with $E_{J}(x, v)<E_{0}$ and consider a characteristic $(X, V)$ going through $(x, v)$, we conclude that if $x \in B_{4}$, we have $X \in B_{4}$ for all time. On the other hand, if $x \notin B_{4}$, we have $X \notin B_{4}$ for all time.

Altogether, assertions (i)-(iii) of the theorem follow, except the non-spherical symmetry in the case $\omega \neq 0$. Choose $x \in \mathbb{R}^{3}$ with $\rho^{\omega}(x)>0, x_{1}:=a \neq 0, x_{2}=x_{3}=0$. Then there exists some $\eta \in \mathbb{R}^{3}$, such that

$$
\frac{1}{2} \eta^{2}+U^{\omega}(x)-\frac{1}{2} \omega^{2} a^{2}<E_{0} .
$$

If $\left(f^{\omega}, U^{\omega}\right)$ were spherically symmetric, there would exist a rotation $A$ around the $x_{2}$-axis such that $(A x)_{1}=(A x)_{2}=0$ and $f^{\omega}(A x, A v)=f^{\omega}(x, v)$. But the monotonicity of $\varphi$ implies

$$
\begin{aligned}
f^{\omega}(x, v) & =\varphi\left(\frac{1}{2} v^{2}+U^{\omega}(x)-\frac{1}{2} \omega^{2} a^{2}\right)=\varphi\left(E_{J}(x, v)\right) \\
& \neq \varphi\left(E_{J}(A x, A v)\right)=\varphi\left(\frac{1}{2} v^{2}+U^{\omega}(x)\right)=f^{\omega}(A x, A v),
\end{aligned}
$$

which contradicts our assumption of spherical symmetry. With a similar argument, one can also show that the constructed solutions cannot be axially symmetric with respect to any axis in $\mathbb{R}^{3}$ except for the $x_{3}$-axis. Although our deformations only have mirror symmetry with respect to every coordinate plane, which would match a triaxial solution, one still has to show that the obtained $\zeta^{\omega}$ are not axially symmetric with repect to the $x_{3}$-axis, which is the major obstruction to the construction of triaxial solutions.

The asserted continuity properties (iv) can be proved as follows: For $x \in B_{3}$ we have

$$
\left|U^{\omega}(x)-U^{\omega^{\prime}}(x)\right| \leq\left\|U_{0}^{\prime}\right\|_{\infty}\left|g_{\zeta_{\omega}}^{-1}(x)-g_{\zeta_{\omega^{\prime}}}^{-1}(x)\right| \leq C\left\|\zeta_{\omega}-\zeta_{\omega^{\prime}}\right\|_{X} .
$$

By the implicit function theorem, $\zeta^{\omega}$ depends continuously on $\omega$ with respect to the $\|\cdot\|_{X}$-norm and we have $\rho^{\omega}(x)=\tilde{h}\left(\omega, r(x), U^{\omega}(x)\right)$.

Lemma 2.3 implies that $\rho^{\omega}$ is continuous in $\omega$ with respect to $\|\cdot\|_{\infty}$ and

$$
U^{\omega}(x)=-\int_{B_{3}} \frac{\rho^{\omega}(y)}{|x-y|} d y+U_{0}(0)+\int_{B_{3}} \frac{\rho^{\omega}(y)}{|y|} d y, \quad x \in \mathbb{R}^{3}
$$

implies the continuity of $U^{\omega}$ in $\omega$ with respect to $\|\cdot\|_{1, \infty}$. Differentiating the above expression for $\rho^{\omega}$ yields the continuity of $\rho^{\omega}$ with respect to $\|\cdot\|_{1, \infty}$ and therefore also the continuity of $U^{\omega}$ in the norm $\|\cdot\|_{2, \infty}$.

3. $\partial_{\zeta} T(0,0)$ is an isomorphism

In this section, we want to establish some of the assumptions needed for the implicit function theorem. We will prove the following result:

Proposition 3.1. The mapping $\partial_{\zeta} T(0,0): X \rightarrow Y$ is a linear isomorphism.

Let $\omega_{2}:=\sqrt{\left|E_{1}\right|} / 4$, where $E_{1}$ is defined in Lemma 2.3 and let us recall from $[8$, 
Prop. 3.1] that the Fréchet-derivative of $T:]-\omega_{2}, \omega_{2}[\times \Omega \rightarrow Y$ is given by

$$
\begin{aligned}
& {\left[\partial_{\zeta} T(\omega, \zeta) \Lambda\right](x)=} \\
= & -\int_{B_{3}}\left(\frac{1}{\left|g_{\zeta}(x)-y\right|}-\frac{1}{|y|}\right) \partial_{u} \tilde{h}\left(\omega, r(y), U_{\zeta}(y)\right) \nabla U_{\zeta}(y) \cdot \frac{g_{\zeta}^{-1}(y)}{\left|g_{\zeta}^{-1}(y)\right|} \Lambda\left(g_{\zeta}^{-1}(y)\right) d y \\
& -\int_{B_{3}} \frac{g_{\zeta}(x)-y}{\left|g_{\zeta}(x)-y\right|^{3}} \tilde{h}\left(\omega, r(y), U_{\zeta}(y)\right) d y \cdot \frac{x}{|x|} \Lambda(x), \quad x \in B_{4},
\end{aligned}
$$

where $\omega \in]-\omega_{2}, \omega_{2}\left[, \zeta \in \Omega, \Lambda \in X\right.$, and $U_{\zeta}(y):=U_{0}\left(g_{\zeta}^{-1}(y)\right), y \in B_{3}$

We abbreviate $L_{0} \Lambda:=\partial_{\zeta} T(0,0) \Lambda$ for $\Lambda \in X$. We observe that $g_{0}=$ id and therefore the function $U_{\zeta}$ in (3.1) coincides with the potential $U_{0}$ of the spherically symmetric steady state we started with, if $\zeta=0$. We have

$$
\begin{aligned}
\rho_{0}^{\prime}(|x|) & =\partial_{u} \tilde{h}\left(0, r(x), U_{0}(|x|)\right) U_{0}^{\prime}(|x|) \\
& =\partial_{u} \tilde{h}\left(0, r(x), U_{0}(|x|)\right) \nabla U_{0}(x) \cdot \frac{x}{|x|}, \quad x \in \mathbb{R}^{3} .
\end{aligned}
$$

This implies that

$$
\begin{aligned}
\left(L_{0} \Lambda\right)(x) & =-\int_{B_{3}}\left(\frac{1}{|x-y|}-\frac{1}{|y|}\right) \rho_{0}^{\prime}(|y|) \Lambda(y) d y-\int_{B_{3}} \frac{x-y}{|x-y|^{3}} \rho_{0}(|y|) d y \cdot \frac{x}{|x|} \Lambda(y) \\
& =-U_{0}^{\prime}(|x|) \Lambda(x)-\int_{B_{3}}\left(\frac{1}{|x-y|}-\frac{1}{|y|}\right) \rho_{0}^{\prime}(|y|) \Lambda(y) d y, \quad x \in B_{4}, \Lambda \in X
\end{aligned}
$$

Now let

$$
(K \Lambda)(x):=-\frac{1}{U_{0}^{\prime}(|x|)} \int_{B_{3}}\left(\frac{1}{|x-y|}-\frac{1}{|y|}\right) \rho_{0}^{\prime}(|y|) \Lambda(y) d y, \quad x \in \dot{B}_{4}, \Lambda \in C_{S}\left(B_{4}\right) .
$$

Then we can write

$$
\left(L_{0} \Lambda\right)(x)=-U_{0}^{\prime}(|x|)[(\mathrm{id}-K) \Lambda](x), \quad x \in B_{4}, \Lambda \in X .
$$

In order to prove Proposition 3.1, we need

Lemma 3.2. The linear operator $K: C_{S}\left(B_{4}\right) \rightarrow C_{S}\left(B_{4}\right)$ is compact, where $C_{S}\left(B_{4}\right)$ is equipped with the supremum norm $\|\cdot\|_{\infty}$.

Proof. For $\Lambda \in C_{S}\left(B_{4}\right)$ let

$$
V_{\Lambda}(x):=-\int_{B_{3}} \frac{1}{|x-y|} \rho_{0}^{\prime}(|y|) \Lambda(y) d y, \quad x \in \mathbb{R}^{3} .
$$

Then $V_{\Lambda} \in C^{1}\left(\mathbb{R}^{3}\right), \nabla V_{\Lambda}(0)=0$, and

$$
(K \Lambda)(x)=\frac{1}{U_{0}^{\prime}(|x|)}\left(V_{\Lambda}(x)-V_{\Lambda}(0)\right), \quad x \in \dot{B}_{4} .
$$

Using Lemma 2.2(c), we obtain the estimate

$$
|(K \Lambda)(x)| \leq \frac{1}{C|x|}\left\|\nabla V_{\Lambda}\right\|_{\infty}|x| \leq C\|\Lambda\|_{\infty}, \quad x \in \dot{B}_{4},
$$


where the constant $C$ depends on $\rho_{0}$ and $U_{0}$, but not on $\Lambda$ or $x$. Thus $K$ maps bounded sets into bounded sets. We next show that $K \Lambda$ is Hölder continuous with exponent $1 / 2$, uniformly on bounded sets in $C_{S}\left(B_{4}\right)$. Let $M>0$ and assume $\|\Lambda\|_{\infty} \leq M$. In the following, constants denoted by $C$ depend on $\rho_{0}, U_{0}$ and $M$, but not on $\Lambda$. Obviously, $\rho_{0}^{\prime} \Lambda \in L^{\infty}\left(\mathbb{R}^{3}\right)$ and we deduce from Lemma A.2 the existence of $C>0$ with

$$
\left|\nabla V_{\Lambda}(x)-\nabla V_{\Lambda}\left(x^{\prime}\right)\right| \leq C\left\|\rho_{0}^{\prime} \Lambda\right\|_{\infty}\left|x-x^{\prime}\right|^{1 / 2}, \quad x, x^{\prime} \in B_{4} .
$$

Since $\nabla V_{\Lambda}(0)=0$, the latter implies

$$
\left|\nabla V_{\Lambda}(x)\right| \leq C|x|^{1 / 2}, \quad x \in B_{4} .
$$

Now let $x, x^{\prime} \in \dot{B}_{4}$ and $|x| \leq\left|x^{\prime}\right|$. Then

$$
\begin{aligned}
\left|(K \Lambda)(x)-(K \Lambda)\left(x^{\prime}\right)\right| \leq & \left|\frac{1}{U_{0}^{\prime}(|x|)}-\frac{1}{U_{0}^{\prime}\left(\left|x^{\prime}\right|\right)}\right|\left|V_{\Lambda}(x)-V_{\Lambda}(0)\right| \\
& +\frac{1}{U_{0}^{\prime}\left(\left|x^{\prime}\right|\right)}\left|V_{\Lambda}(x)-V_{\Lambda}\left(x^{\prime}\right)\right|=: I_{1}+I_{2}
\end{aligned}
$$

and we obtain for some $z \in B_{4}$ with $|z| \leq\left|x^{\prime}\right|$ the estimates

$$
\begin{aligned}
I_{1} & \leq \frac{\left|U_{0}^{\prime}(|x|)-U_{0}^{\prime}\left(\left|x^{\prime}\right|\right)\right|}{|x|\left|x^{\prime}\right|}\left|\nabla V_{\Lambda}(z)\right||x| \leq C\left|x-x^{\prime}\right|^{1 / 2} \frac{\left(|x|+\left|x^{\prime}\right|\right)^{1 / 2}}{\left|x^{\prime}\right|}|z|^{1 / 2} \\
& \leq C\left|x-x^{\prime}\right|^{1 / 2}
\end{aligned}
$$

and

$$
I_{2} \leq \frac{C}{\left|x^{\prime}\right|}\left|\nabla V_{\Lambda}(z)\right|\left|x-x^{\prime}\right| \leq \frac{C}{\left|x^{\prime}\right|}|z|^{1 / 2}\left|x-x^{\prime}\right| \leq C\left|x-x^{\prime}\right|^{1 / 2},
$$

so that

$$
\left.\mid\left(K_{\Lambda}\right)(x) K_{\Lambda}\right)\left(x^{\prime}\right)|\leq C| x-\left.x^{\prime}\right|^{1 / 2}, \quad x, x^{\prime} \in \dot{B}_{4}
$$

and

$$
\left|\left(K_{\Lambda}\right)(x)\right| \leq C\left|\nabla V_{\Lambda}(z)\right| \leq C|x|^{1 / 2}, \quad x \in \dot{B}_{4} .
$$

We have shown that $K$ maps bounded sets of $C_{S}\left(B_{4}\right)$ into bounded and equicontinuous subsets of $C_{S}\left(B_{4}\right)$. Thus $K$ is compact by the Arzela-Ascoli theorem and the proof is complete.

Lemma 3.3. id $-K: C_{S}\left(B_{4}\right) \rightarrow C_{S}\left(B_{4}\right)$ is one-to-one and onto.

Proof. Since $K$ is compact, it suffices to show that id $-K$ is one-to-one. Let $\Lambda \in C_{S}\left(B_{4}\right)$ with $\Lambda-K \Lambda=0$. Now $\Lambda=0$ can be shown by expanding $\Lambda$ into spherical harmonics. For that purpose, let

$$
\left\{\mathcal{S}_{n, j}, n \in \mathbb{N}, j=1, \ldots, 2 n+1\right\}
$$

be the orthonormal set of spherical harmonics introduced in the Appendix, where for $n \in \mathbb{N}$, the functions $\mathcal{S}_{n, j}: \partial B_{1} \rightarrow \mathbb{R}, j=1, \ldots, 2 n+1$ are homogeneous polynomials of degree $n$. We define

$$
\Lambda_{n j}(r):=\int_{\partial B_{1}} \mathcal{S}_{n, j}(\xi) \Lambda(r \xi) d \omega_{\xi}=\frac{1}{r^{2}} \int_{\partial B_{r}} \mathcal{S}_{n, j}(x / r) \Lambda(x) d \omega_{x}
$$


and we use the expansion of the integral kernel $1 /|x-y|$ into spherical harmonics from Lemma A.3 and Lemma A.4: For $x, y \in \mathbb{R}^{3}, x=r \xi$ and $y=s \eta$ with $\xi, \eta \in \partial B_{1}, r, s \in \mathbb{R}^{+}$, $r \neq s$, we have

$$
\frac{1}{|x-y|}=\max (r, s)^{-1} \sum_{n=0}^{\infty} \sum_{j=1}^{2 n+1} \frac{4 \pi}{2 n+1}\left(\frac{\min (r, s)}{\max (r, s)}\right)^{n} \mathcal{S}_{n, j}(\xi) \mathcal{S}_{n, j}(\eta)
$$

$K \Lambda-\Lambda=0$ then implies

$$
\begin{aligned}
\Lambda_{n j}(r)=- & \frac{1}{U_{0}^{\prime}(r)} \int_{B_{3}} \int_{\partial B_{1}}\left(\frac{1}{|r \xi-y|}-\frac{1}{|y|}\right) \mathcal{S}_{n, j}(\xi) d \omega_{\xi} \rho_{0}^{\prime}(|y|) \Lambda(y) d y \\
=- & \frac{4 \pi}{2 n+1} \frac{1}{U_{0}^{\prime}(r)} \int_{0}^{3} s^{2} \rho_{0}^{\prime}(s) \frac{\min (r, s)^{n}}{\max (r, s)^{n+1}} \int_{\partial B_{1}} \mathcal{S}_{n, j}(\eta) \Lambda(s \eta) d \omega_{\eta} d s \\
& \quad+\frac{4 \pi}{2 n+1} \frac{1}{U_{0}^{\prime}(r)} \int_{0}^{3} s^{2} \rho_{0}^{\prime}(s) \frac{0^{n}}{s^{n+1}} \int_{\partial B_{1}} \mathcal{S}_{n, j}(\eta) \Lambda(s \eta) d \omega_{\eta} d s \\
=- & \frac{4 \pi}{2 n+1} \frac{1}{U_{0}^{\prime}(r)} \int_{0}^{3} s^{2} \rho_{0}^{\prime}(s)\left(\frac{\min (r, s)^{n}}{\max (r, s)^{n+1}}-\frac{0^{n}}{s^{n+1}}\right) \Lambda_{n j}(s) d s,
\end{aligned}
$$

where we used that the functions $\mathcal{S}_{n, j}$ are orthonormal with repsect to $\langle\cdot, \cdot\rangle_{L^{2}\left(\partial B_{1}\right)}$. We find that

$$
\Lambda_{01}(r)=-\frac{4 \pi}{r U_{0}^{\prime}(r)} \int_{0}^{r} \rho_{0}^{\prime}(s) s(s-r) \Lambda_{01}(s) d s
$$

and we obviously have $\lim _{r \rightarrow 0} \Lambda_{01}(r)=0$. Let $R \geq 0$ be maximal such that $\Lambda_{01}(r)$ vanishes on $[0, R]$. Then for $r \in[R, 3]$,

$$
\left|\Lambda_{01}(r)\right| \leq \frac{4 \pi}{r U_{0}^{\prime}(r)}\left\|\rho_{0}^{\prime}\right\|_{\infty} \sup _{0 \leq s \leq r}\left|\Lambda_{01}(s)\right| \int_{R}^{r} s(r-s) d s \leq C(r-R) \sup _{0 \leq s \leq r}\left|\Lambda_{01}(s)\right| .
$$

Thus for small $\epsilon>0$, we have $\Lambda_{01}(r)=0$ on the interval $[R, R+\epsilon]$ and we conclude that $\Lambda_{01}$ vanishes on the whole interval $[0,3]$. Now up to linear combinations, the spherical harmonics for $n=1$ are given by $x_{1}, x_{2}, x_{3}$, and $\Lambda \in C_{S}$ implies

$$
\int_{\partial B_{1}} \xi_{1} \Lambda(r \xi) d \omega_{\xi}=-\int_{\partial B_{1}} \xi_{1} \Lambda(r \xi) d \omega_{\xi}=0
$$

where we made the transformation $\xi \mapsto\left(-\xi_{1}, \xi_{2}, \xi_{3}\right)$. Analoguously,

$$
\int_{\partial B_{1}} \xi_{2} \Lambda(r \xi) d \omega_{\xi}=\int_{\partial B_{1}} \xi_{3} \Lambda(r \xi) d \omega_{\xi}=0
$$

and we have $\Lambda_{11}=\Lambda_{12}=\Lambda_{13} \equiv 0$. Let $n \geq 2$. Then

$$
\Lambda_{n j}(r)=-\frac{4 \pi}{2 n+1} \frac{1}{U_{0}^{\prime}(r)}\left(\int_{0}^{r} s^{2} \rho_{0}^{\prime}(s) \frac{s^{n}}{r^{n+1}} \Lambda_{n j}(s) d s+\int_{r}^{3} s^{2} \rho_{0}^{\prime}(s) \frac{r^{n}}{s^{n+1}} \Lambda_{n j}(s) d s\right)
$$


and

$$
\begin{aligned}
\left|\Lambda_{n j}(r)\right| & \leq \frac{4 \pi}{2 n+1} \frac{1}{U_{0}^{\prime}(r)}\left\|\Lambda_{n j}\right\|_{\infty}\left(\frac{1}{r^{2}} \int_{0}^{r}\left(-\rho_{0}^{\prime}\right)(s) \frac{s^{n-1}}{r^{n-1}} s^{3} d s+r \int_{r}^{3}\left(-\rho_{0}^{\prime}\right)(s) \frac{r^{n-1}}{s^{n-1}} d s\right) \\
& \leq \frac{4 \pi}{2 n+1} \frac{1}{U_{0}^{\prime}(r)}\left\|\Lambda_{n j}\right\|_{\infty}\left(\frac{1}{r^{2}} \int_{0}^{r}\left(-\rho_{0}^{\prime}\right)(s) s^{3} d s+r \int_{r}^{3}\left(-\rho_{0}^{\prime}\right)(s) d s\right) \\
& =\frac{4 \pi}{2 n+1} \frac{1}{U_{0}^{\prime}(r)}\left\|\Lambda_{n j}\right\|_{\infty}\left(\frac{1}{r^{2}} r^{3}\left(-\rho_{0}\right)(r)+\frac{3}{r^{2}} \int_{0}^{r} s^{2} \rho_{0}(s) d s+r \rho_{0}(r)\right) \\
& =\frac{3}{2 n+1}\left\|\Lambda_{n j}\right\|_{\infty},
\end{aligned}
$$

where we integrated by parts in the third line and used the fact that $U_{0}^{\prime}(r)=$ $\frac{4 \pi}{r^{2}} \int_{0}^{r} s^{2} \rho_{0}(s) d s$ in the last line, also recalling from $(2.2)$ that $-\rho_{0}^{\prime}(r) \geq 0$.

Now $2 n+1>3$ for $n \geq 2$ implies that $\Lambda_{n j} \equiv 0$ for $n \geq 2$ as well and the completeness of $\left\{\mathcal{S}_{n, j}\right\}$ induces $\Lambda \equiv 0$. We conclude that id $-K$ is one-to-one as claimed.

It is now clear that $L_{0}: X \rightarrow Y$ is one-to-one as well - this follows from equation (3.2) and the fact that $U_{0}^{\prime}(r)>0$ for $r>0$. Once we have proved the following lemma, the proof of Proposition 3.1 will be complete.

LEMMA 3.4. $L_{0}: X \rightarrow Y$ is onto.

Proof. Let $g \in Y$ and define $q:=g / U_{0}^{\prime}$. We will show $q \in X$. We have that $q \in$ $C^{1}\left(\dot{B}_{4}\right) \cap C_{S}\left(B_{4}\right)$ and

$$
|\nabla q| \leq \frac{|\nabla g(x)|}{U_{0}^{\prime}(|x|)}+|g(x)|\left|\frac{U_{0}^{\prime \prime}(|x|)}{U_{0}^{\prime}(|x|)^{2}} \frac{x}{|x|}\right| \leq C\left(\frac{\mid \nabla g(x)}{|x|}+\frac{|g(x)|}{|x|^{2}}\right) \leq 2 C\|g\|_{Y} .
$$

By definition of $Y$ and since $U_{0} \in C^{2}\left(\left[0, \infty[)\right.\right.$ with $U_{0}^{\prime \prime}(0)>0$, we have that for every $x \in \partial B_{1}$,

$$
\begin{aligned}
\nabla q(t x) & =\frac{\nabla g(t x)}{t} \frac{t}{U_{0}^{\prime}(t)}-\frac{g(t x)}{t^{2}} U_{0}^{\prime \prime}(t)\left(\frac{t}{U_{0}^{\prime}(t)}\right)^{2} x \\
& \rightarrow \frac{\nabla g(0 x)}{0} \frac{1}{U_{0}^{\prime \prime}(0)}-\frac{g(0 x)}{0^{2}} U_{0}^{\prime \prime}(0) \frac{1}{U_{0}^{\prime \prime}(0)^{2}} x
\end{aligned}
$$

as $t \rightarrow 0+$, uniformly in $x \in \partial B_{1}$.

Since $X \subset C_{S}\left(B_{4}\right)$, there exists by Lemma 3.3 an element $\Lambda \in C_{S}\left(B_{4}\right)$ such that

$$
\Lambda-K \Lambda=-q=-\frac{g}{U_{0}^{\prime}}
$$

This implies that $L_{0} \Lambda=g$ and thus that $L_{0}$ is onto, provided $\Lambda \in X$. To see the latter statement, we observe that $\Lambda=K \Lambda-q$ is Hölder continuous since $K \Lambda$ is Hölder continuous. If we now define $V_{\Lambda}$ as above in the proof of Lemma 3.2 we also conclude that $V_{\Lambda} \in C^{2}\left(\mathbb{R}^{3}\right)$ and thus $K \Lambda \in C^{1}\left(\dot{B}_{4}\right)$. Denoting by $H_{V_{\Lambda}}$ the Hessian of $V_{\Lambda}$, we obtain for each $x \in \dot{B}_{4}$ a point $z \in \overline{0, x}$ such that

$$
\begin{aligned}
|\nabla(K \Lambda)(x)| & \leq\left|\frac{U_{0}^{\prime \prime}(|x|)}{U_{0}^{\prime}(|x|)^{2}}\right|\left|V_{\Lambda}(x)-V_{\Lambda}(0)\right|+\frac{1}{\left|U_{0}^{\prime}(|x|)\right|}\left|\nabla V_{\Lambda}(x)\right| \\
& \leq \frac{C}{|x|^{2}}\left|\left\langle H_{V_{\Lambda}}(z) x, x\right\rangle\right|+\frac{C}{|x|}\left|\nabla V_{\Lambda}(x)\right| \leq C\left\|D^{2} V_{\Lambda}\right\|_{\infty},
\end{aligned}
$$


where we defined $\langle x, y\rangle:=\sum_{j=1}^{3} x_{j} y_{j}$ for $x, y \in \mathbb{R}^{3}$. Finally, for $x \in \partial B_{1}$, we have

$$
\begin{aligned}
\nabla(K \Lambda)(t x) & =-\frac{U_{0}^{\prime \prime}(t)}{U_{0}^{\prime}(t)^{2}} x\left(V_{\Lambda}(t x)-V_{\Lambda}(0)\right)+\frac{1}{U_{0}^{\prime}(t)} \nabla V_{\Lambda}(t x) \\
& =-U_{0}^{\prime \prime}(t)\left(\frac{t}{U_{0}^{\prime}(t)}\right)^{2} x \frac{1}{t^{2}} \frac{1}{2}\left\langle H_{V_{\Lambda}}(\tau x) t x, t x\right\rangle+\frac{t}{U_{0}^{\prime}(t)} \frac{\nabla V_{\Lambda}(t x)}{t} \\
& \rightarrow-\frac{1}{2 U_{0}^{\prime \prime}(0)}\left\langle H_{V_{\Lambda}}(0) x, x\right\rangle x+\frac{1}{U_{0}^{\prime \prime}(0)} D^{2} V_{\Lambda}(0) x,
\end{aligned}
$$

as $t \rightarrow 0+$, uniformly in $x \in \partial B_{1}$. We have shown that $K \Lambda \in X$, which implies $\Lambda=$ $K \Lambda+q \in X$, and the proof is complete.

Appendix A. In this section, we first state the implicit function theorem, which is used for the proof of Theorem 2.1. We then give a regularity result for the Poisson equation and finally introduce spherical harmonics. We state two important lemmas: an addition theorem and the expansion of the integral kernel $1 /|x-y|$ in spherical harmonics.

Theorem A.1. Let $X, Y, Z$ be Banach spaces, $U \subset X$ and $V \subset Y$ be neighborhoods of $x_{0} \in X$ and $y_{0} \in Y$, respectively, and $F: U \times V \rightarrow Z$ be continuous and continously Fréchet-differentiable with respect to the second variable. Suppose also that $F\left(x_{0}, y_{0}\right)=$ 0 and $F_{y}^{-1}\left(x_{0}, y_{0}\right) \in \mathfrak{L}(Z, Y)$.

Then there exist balls $\overline{B_{r}}\left(x_{0}\right) \subset U, \overline{B_{\delta}}\left(y_{0}\right) \subset V$ and exactly one continuous map $G: B_{r}\left(x_{0}\right) \rightarrow B_{\delta}\left(y_{0}\right)$ such that $G x_{0}=y_{0}$ and $F(x, G x)=0$ on $B_{r}\left(x_{0}\right)$.

Proof. [1, Thm. 15.1].

Lemma A.2. Let $n<p \leq \infty$ and let $\rho(x) \in L^{p}\left(\mathbb{R}^{n}\right)$ with compact support. Define

$$
V_{\rho}(x):=-\int_{\mathbb{R}^{n}} \frac{1}{|x-y|} \rho(y) d y .
$$

Then for every $0<\alpha<1-n / p$ we have $V_{\rho} \in C^{1, \alpha}\left(\mathbb{R}^{n}\right)$ and

$$
\left|\partial_{i} V_{\rho}(x)-\partial_{i} V_{\rho}\left(x^{\prime}\right)\right| \leq C(n, \alpha, p)\left|x^{\prime}-x\right|^{\alpha}\|f\|_{p} \mathcal{L}^{n}(\operatorname{supp}\{\rho\})^{\frac{1-\alpha}{n}-\frac{1}{p}} .
$$

Proof. [6, Thm. 10.2].

Some facts about spherical harmonics. In the following, we use the notation of [7] and we will always consider the case where the spatial dimension $q$ is equal to 3 . For $n \in \mathbb{N}$, consider a homogeneous polynomial $H_{n}$ of degree $n$, which satisfies

$$
\Delta H_{n}(x)=0 .
$$

Then for $\xi \in \partial B_{1}:=\left\{x \in \mathbb{R}^{3}|| x \mid=1\right\}$,

$$
S_{n}(\xi):=H_{n}(\xi)
$$

is called a spherical harmonic of order $n$. For each $n$, there exist $2 n+1$ linearly independent spherical harmonics, which we call $S_{n, j}, j=1, \ldots 2 n+1$ [7, Lem. 4]. We denote by $\left\{\mathcal{S}_{n, j}, \quad n=0, \ldots, \infty, j=1, \ldots, 2 n+1\right\}$ the orthonormal set of all spherical harmonics, where the orthonormalization is done with respect to $\langle., .\rangle_{L^{2}\left(\partial B_{1}\right)}$. Then we have the following 
Lemma A.3. For a fixed $n \in \mathbb{N}$ and $\xi, \eta \in \partial B_{1}$, we have that

$$
\sum_{j=1}^{2 n+1} \mathcal{S}_{n, j}(\xi) \mathcal{S}_{n, j}(\eta)=\frac{2 n+1}{4 \pi} P_{n}(\xi \cdot \eta),
$$

where $P_{n}(t)$ is the Legendre Polynomial of degree $n$.

Lemma A.4. Let $x, y \in \mathbb{R}^{3}$ with $x=R \xi, y=r \eta$, for suitable $\xi, \eta \in \partial B_{1}$ and $r, R \in \mathbb{R}$. Then we have for $R>r$

$$
\frac{1}{|x-y|}=R^{-1} \sum_{n=0}^{\infty}\left(\frac{r}{R}\right)^{n} P_{n}(\xi \cdot \eta)
$$

and for $R<r$

$$
\frac{1}{|x-y|}=r^{-1} \sum_{n=0}^{\infty}\left(\frac{R}{r}\right)^{n} P_{n}(\xi \cdot \eta)
$$

where $P_{n}(t)$ is the Legendre Polynomial of degree $n$.

Proofs can be found in [7, Thm. 2 and Lem. 19].

Acknowledgements. The author wishes to thank Gerhard Rein for the critical review of the manuscript. This research was supported by the Deutsche Forschungsgemeinschaft under the project "Nichtlineare Stabilität bei kinetischen Modellen aus der Astrophysik und Plasmaphysik".

\section{REFERENCES}

[1] K. Deimling, Nonlinear Functional Analysis, Springer, Berlin, 1985.

[2] B. Gidas, W.-M Ni and L. Nirenberg, Symmetry and related properties via the maximum principle, Comm. Math. Phys., 68, 209-243, 1979.

[3] G. Heilig, On Lichtenstein's analysis of rotating Newtonian stars, Ann. Inst. Henri Poincaré, Physique théorique, 60, 457-487, 1994.

[4] L. Lichtenstein, Untersuchung über die Gleichgewichtsfiguren rotierender Flüssigkeiten, deren Teilchen einander nach dem Newtonschen Gesetze anziehen. Erste Abhandlung. Homogene Flüssigkeiten. Allgemeine Existenzsätze, Math. Z., 1, 229-284, 1918.

[5] L. Lichtenstein, Gleichgewichtsfiguren Rotierender Flüssigkeiten, Springer, Berlin, 1933.

[6] E. H. Lieb and M. Loss, Analysis, Amer. Math. Soc., Providence, RI, 2001.

[7] C. Müller, Spherical Harmonics, Lecture Notes in Mathematics 17, Springer, Berlin, 1966.

[8] G. Rein, Stationary and static stellar dynamic models with axial symmetry, Nonlinear Anal., 41, 313-344, 2000.

[9] P.O. Vandervoort, The nonaxisymmetric configurations of uniformly rotating polytropes, Astrophys. J., 241, 316-333, 1980. 